\title{
The effects of glutamine on intestinal epithelial cell proliferation in parenterally fed rats
}

\author{
N Mandir, R A Goodlad
}

\begin{abstract}
Background-Several papers have indicated that glutamine is a preferred fuel for the enterocyte and that it can increase intestinal epithelial cell proliferation. Aims-To investigate the effects of glutamine on intestinal epithelial cell proliferation in the parenterally fed rat.

Methods-Five groups of six rats were fed parenterally; a group of orally fed rats was also studied. Crypt cell proliferation was studied after six days using native mitoses in microdissected crypts and bromodeoxyuridine labelling.

Results-No effect of treatment was seen on intestinal weight; however, the weights of the small intestine, caecum, and colon were all significantly heavier in the orally fed group than in the total parenteral nutrition groups $(p<0.001)$. There was no effect of any of the glutamine treatments on mitotic activity in the small intestine. In the colon there was a small increase in native mitoses with glutamine $(p=0.03)$. There was also an indication of increased proliferative activity in the first fifth of the small intestine and colon with glutamine. Little effect of glutamine on bromodeoxyuridine labelling in either site was observed, but there was a small but significant reduction in growth fraction of the colon of the glutamine treated group. The labelling distribution curve from sections and the mitotic distribution curve obtained from crypt squashes showed a good correlation.

Conclusion-Glutamine has a small, but significant effect on mitotic activity but only in the colon. Modest effects on the distribution of labelled cells were also seen.

(Gut 1999;44:608-614)
\end{abstract}

Department of Histopathology, Imperial College School of Medicine, London, UK R Goodlad

Histopathology Unit, Imperial Cancer Research Fund, 44 Lincoln's Inn Fields, London WC2A 3PX, UK

R Mandir

R A Goodlad

Correspondence to: Dr Goodlad.

Accepted for publication 18 November 1998
Keywords: glutamine; parenteral nutrition; cell proliferation; intestine

In addition to its role in digestion and absorption the gastrointestinal tract is also a multilayered defence system One of the mainstays for the maintenance of the integrity of this system is epithelial cell proliferation. The control of gastrointestinal epithelial cell proliferation is multifactorial, with the presence or absence of food in the intestinal lumen being one of the most powerful stimuli for mucosal cell proliferation. The actions of food in the lumen can either be a direct response (luminal nutrition) or a response to the work of absorption (intestinal workload). Various nutrients appear able to stimulate intestinal epithelial cell proliferation and although they may act locally, actions can also be moderated by systemic factors. Luminal nutrition is virtually abolished and the gut is almost entirely dependent on blood borne nutrients when intravenous feeding is commenced. A profound intestinal atrophy is then seen in the animal ${ }^{1}$ and in man. ${ }^{2}$ There is a considerable body of evidence suggesting that the gut of these TPN animals is at risk of becoming deficient in the amino acid glutamine, which although defined as nonessential, is one of the preferred respiratory fuels for enterocytes. ${ }^{34}$ There are several reports of beneficial effects of additional glutamine, ${ }^{56}$ and many of these have reported improved intestinal morphology. ${ }^{7}$ There is some confusion in the literature concerning this, as other reports have found no change in the mucosa when parenteral nutrition was supplemented with glutamine. ${ }^{8}$ Some of the confusion can be attributable to the choice of measured endpoint, as many studies have focused on clinical outcome or resistance to bacterial translocation rather than proliferation per se. Several different models of atrophy have also been used; thus glutamine enriched elemental diet in a model of radiation induced damage led to significant increases in jejunal villous number, villous height, and number of metaphase mitoses per crypt $^{9}$ and following small bowel allotransplantation. ${ }^{10}$ Nonetheless no effect of glutamine was reported after bowel resection. ${ }^{11}$ There are even reports of reduced adaptation following (high) doses of glutamine. $^{12}$

We therefore used our TPN feeding system to investigate the effects of adding glutamine on intestinal cell kinetics at various sites throughout the gut, using quantification of mitotic figures in crypt squashes ${ }^{13}$ and bromodeoxyuridine labelled DNA synthesising cells. ${ }^{14}$ These data were analysed to determine whether there were any changes in cell production or in the distribution of dividing cells within the crypt. We also used alanine as an "inert" amino acid to balance the addition of extra nitrogen and energy from glutamine. In addition we took the opportunity to compare the labelling date with that obtained from the quantification of native mitoses.

Materials and methods

Six groups of male Wistar rats, mean weight $250 \mathrm{~g}$, were used with six rats per group. Five groups of rats were infused with a total

Abbreviations used in this paper: BrdU, bromodeoxyuridine; Gln, glutamine; TPN, total parenteral nutrition. 

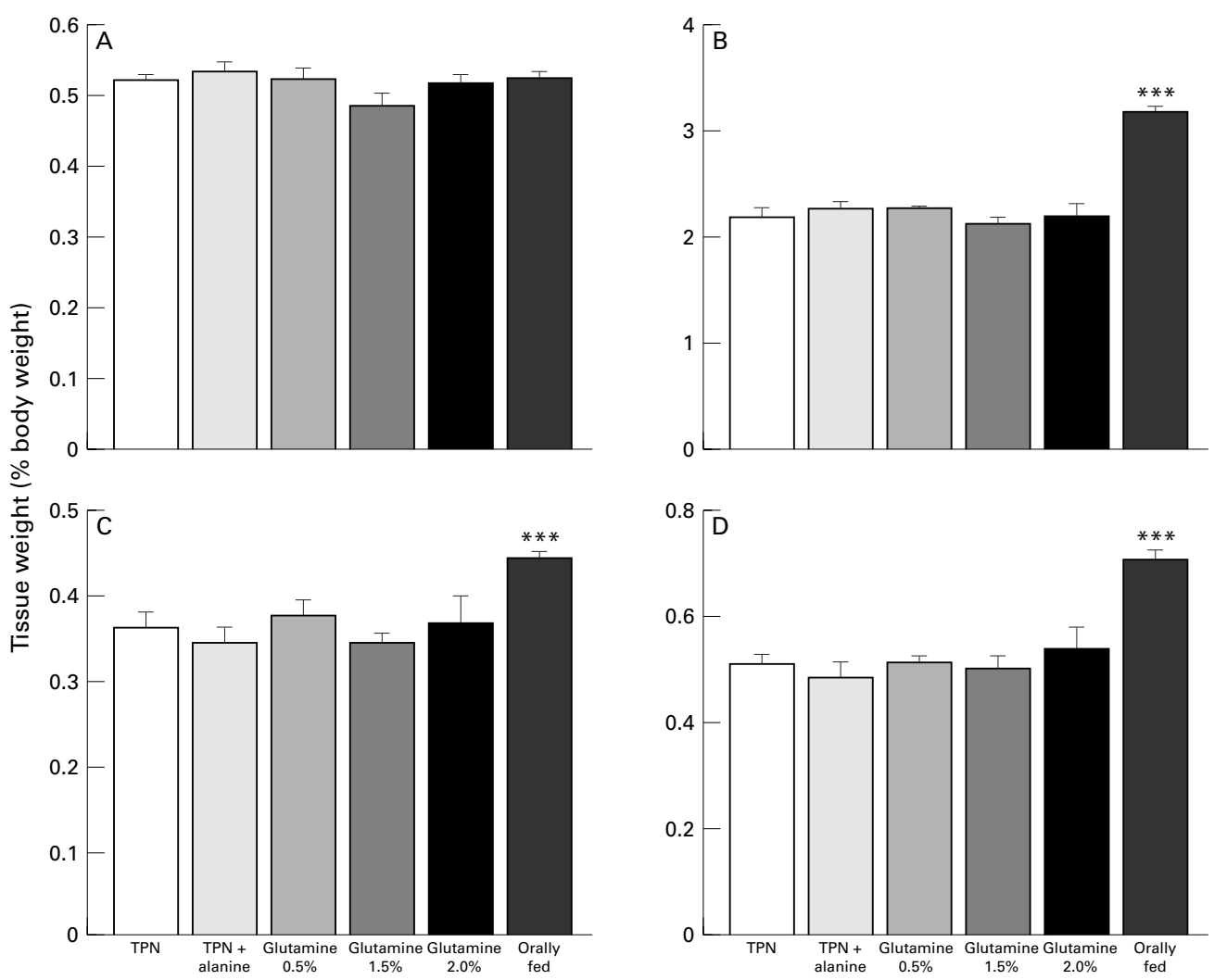

Figure 1 The weights of the stomach (A), small intestine (B), caecum (C), and colon (D), expressed as a percentage of total body weight. ${ }^{\star \star \star} p<0.001$ versus $T P N+$ alanine.

parenteral nutrition (TPN) diet for six days. The first group was infused with the basic TPN diet, the second was given TPN supplemented with $2 \%$ alanine. There was no difference between the TPN and the TPN + alanine groups; the latter was the control group. The third, fourth, and fifth groups were given TPN supplemented with $0.5 \%, 1.5 \%$, and $2 \%$ glutamine. The diets were made up in a laminar air cabinet using aseptic techniques. The glutamine was dissolved in the amino acid electrolyte solution and sterilised by filtration using Millipore filters. The sixth group of rats was fed orally.

All procedures were approved by the Imperial Cancer Research Fund Animal Ethics Committee.

All animals were given a combination anaesthetic consisting of $0.10 \mathrm{ml}$ of Hypnorm intramuscularly (Janssen Animal Health), and diazepam intraperitoneally (Phoenix Pharmaceuticals Limited, Gloucester, UK). For the TPN groups a silastic cannula was inserted and tied into the right external jugular vein, tunnelled subcutaneously to the back of the neck, threaded through a stainless steel skin button, and connected to an Instech fluid swivel joint (Linton Instrumentation, Norfolk, UK). The rats were housed individually in wire bottomed cages with free access to water. The refrigerated TPN diet was pumped into the rats by a multichannel peristaltic pump, at a rate of $60 \mathrm{ml} /$ rat/day. ${ }^{15}$

Rats were sacrificed at intervals of 15 minutes on the sixth day postoperation. This occurred one hour after administration of 50 $\mathrm{mg} / \mathrm{kg}$ bromodeoxyuridine (BrdU; Sigma Chemicals, Poole, Dorset, UK), given intravenously in the TPN groups and intraperitoneally in the orally fed animals. Terminal anaesthesia was induced by sodium pentobarbitone injection. Samples of the small intestine and colon, defined by their percentage length, were fixed in Carnoy's fluid for three hours and then transferred to and stored in $70 \%$ ethanol. For the microdissection method a small piece of the tissue was later stained by the Feulgen reaction, gently teased apart, and the number of native mitoses in 20 intestinal crypts or colonic crypts scored. Further tissue was used to determine the distribution of mitotic figures using a drawing tube to project an image of a five zone grid into the field of view of the microscope. The size of the grid was altered by raising or lowering it using a laboratory scissor jack; this allowed alteration of the grid size to fit the crypt being studied and "standardisation" of the crypts at the same time. Forty crypts per animal were scored.

For histological analysis the mucosa of the TPN control, high dose glutamine, and orally fed rats was embedded in wax, oriented perpendicular to the surface, and alternate $4 \mu \mathrm{m}$ sections were cut and mounted. Immunohistochemistry was performed by the streptavidin biotin conjugate method (SABC) using a mouse monoclonal antibody to BrdU; the second antibody was rabbit antimouse biotin conjugated, and the third layer was a streptavidin peroxidase complex, which was developed in 3',3diaminobenzidine tetrahydrochloride (DAB) 

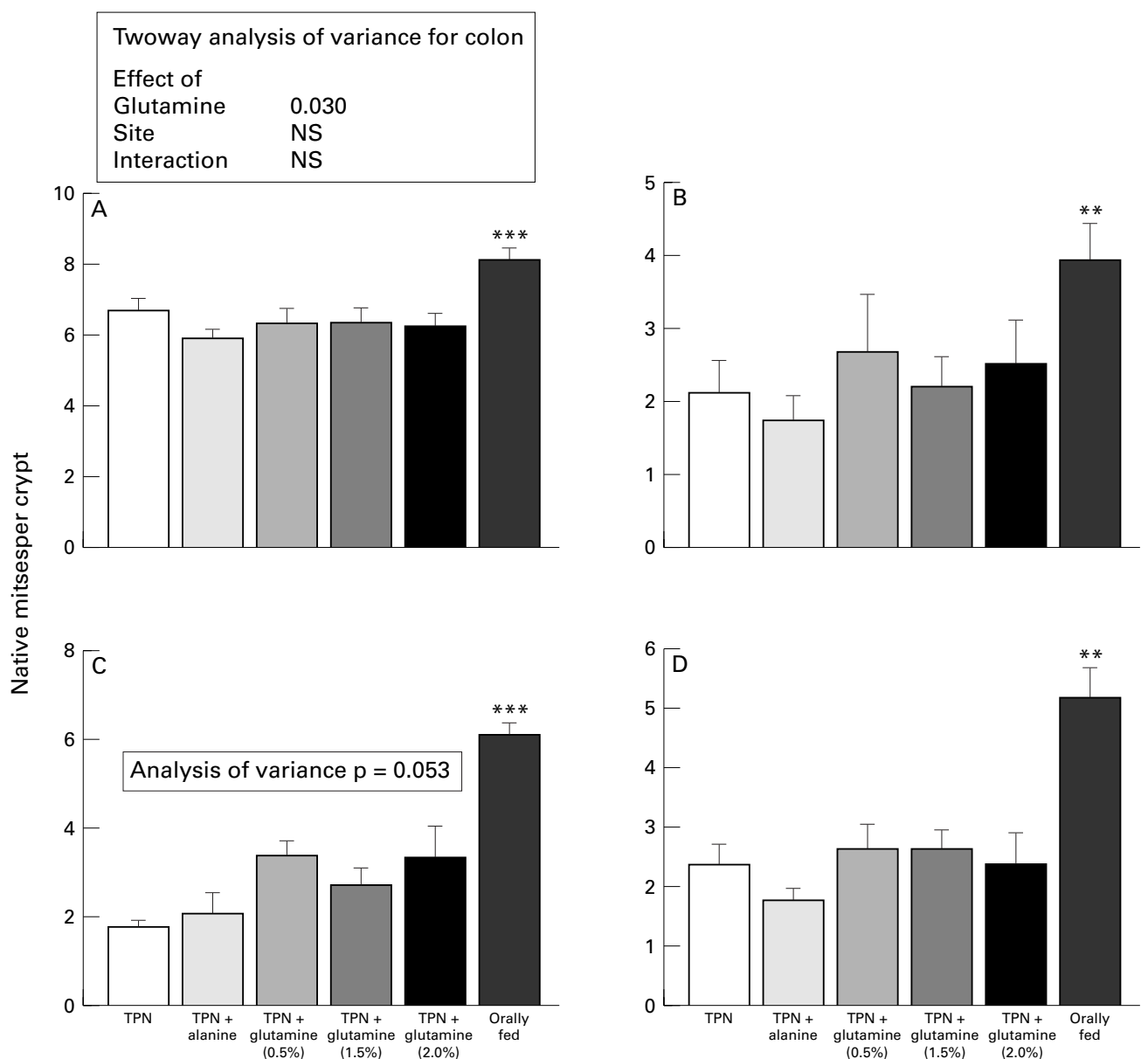

Figure 2 Native mitoses counts in crypt squash preparations in the small intestine and colon: (A) 50\% small intestine; (B) $10 \%$ colon; (C) $50 \%$ colon; (D) $90 \%$ colon. ${ }^{\star *} p<0.01,{ }^{\star \star *} p<0.001$ versus $T P N+$ alanine.

plus hydrogen peroxide ${ }^{16}$ and counterstained with haematoxylin.

Slides were examined systematically until well oriented crypts were found. The crypt was then scored, from the crypt base (position 0 ) to the crypt villus junction or the top of the colonic crypt. The presence and location of labelled and mitotic cells and the crypt length were recorded. The product of the length (col- umn count) and the crypt diameter (cell count) gave the crypt cell population. ${ }^{17}$ Thirty crypts per animal were scored. The labelling and mitotic data were computed to determine the maximum and the half maximum labelling position. ${ }^{14}$ The crypts were also standardisedthat is, the distribution data per crypt were adjusted to the average crypt length per rat, and the distribution of labelling in five equal zones

Table 1 Mitotic and labelled cells in sections of the small intestine and colon

\begin{tabular}{|c|c|c|c|c|c|c|}
\hline & \multicolumn{2}{|l|}{$T P N$} & \multicolumn{2}{|c|}{$T P N+$ glutamine } & \multicolumn{2}{|c|}{ Orally fed } \\
\hline & Mean & SEM & Mean & SEM & Mean & SEM \\
\hline \multicolumn{7}{|l|}{ Small intestine } \\
\hline Number of mitoses per crypt column & 1.24 & 0.26 & 1.09 & 0.19 & 1.41 & 0.11 \\
\hline Mitotic index $(\%)$ & 3.11 & 0.52 & 2.84 & 0.43 & 3.24 & $0.30^{\star}$ \\
\hline Number of labelled cells per crypt column & 9.10 & 0.35 & 8.25 & 0.54 & 10.21 & 0.37 \\
\hline Labelling index $(\%)$ & 23.47 & 0.94 & 22.22 & 0.58 & 23.32 & 0.72 \\
\hline Crypt column length (cells) & 39.32 & 1.90 & 37.16 & 1.55 & 44.28 & $0.73^{\star}$ \\
\hline Maximum labelling position (cell position) & 14.64 & 0.58 & 13.38 & 0.70 & 13.18 & 1.08 \\
\hline $50 \%$ labelling position (cell position) & 20.92 & 0.66 & 19.89 & 0.90 & 19.76 & 1.47 \\
\hline Growth fraction & 0.54 & 0.02 & 0.54 & 0.01 & 0.45 & $0.03^{\star}$ \\
\hline \multicolumn{7}{|l|}{ Colon } \\
\hline Number of mitoses per crypt column & 0.33 & 0.12 & 0.55 & 0.17 & 0.62 & $0.63^{0.06}$ \\
\hline Mitotic index $(\%)$ & 0.70 & 0.26 & 1.11 & 0.31 & 1.24 & 0.14 \\
\hline Number of labelled cells per crypt column & 4.61 & 0.60 & 5.49 & 0.80 & 5.13 & 0.34 \\
\hline Labelling index $(\%)$ & 9.90 & 1.39 & 11.26 & 1.39 & 10.42 & 0.55 \\
\hline Crypt column length (cells) & 48.10 & 1.04 & 48.50 & 2.37 & 49.42 & 1.24 \\
\hline Maximum labelling position (cell position) & 15.03 & 0.46 & 13.84 & 0.71 & 15.44 & 0.85 \\
\hline $50 \%$ labelling position (cell position) & 20.93 & 0.57 & 19.39 & 1.00 & 21.47 & 0.98 \\
\hline Growth fraction & 0.46 & 0.02 & 0.40 & $0.02^{\star}$ & 0.44 & 0.02 \\
\hline
\end{tabular}

Results expressed per crypt column and as indexes. The distribution of labelled cells per column was used to calculate the position of maximum labelling and half maximum labelling and thus define the growth fraction. Statistics refer to comparisons with the TPN control groups. ${ }^{\star} \mathrm{p}<0.05 ;{ }^{0.06}=$ probability. 

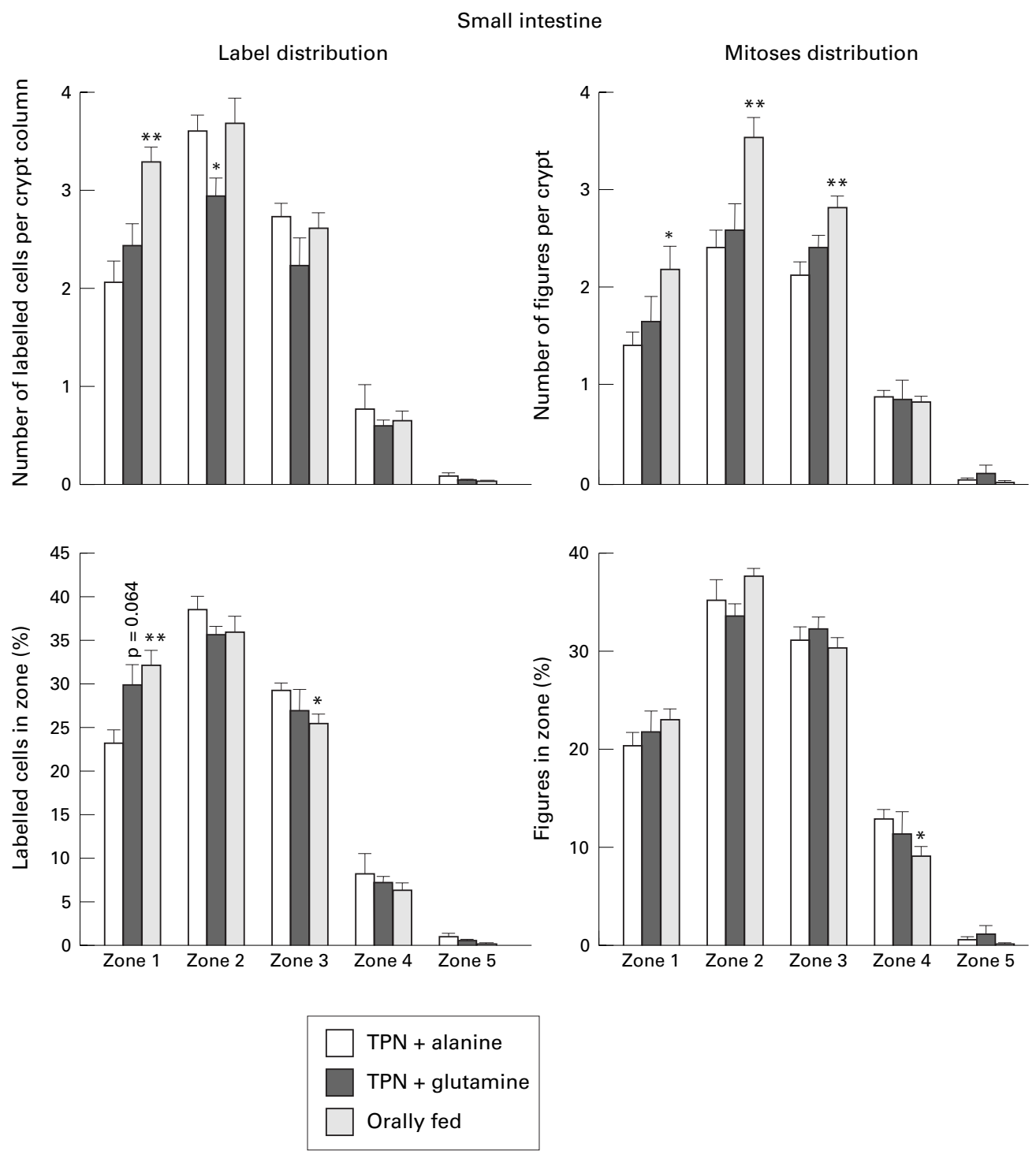

Figure 3 Data from sections reformatted to express the proportion of labelled cells within five equal zones of the crypt and the distribution of mitotic figures scored by superimposing a grid over the individual microdissected crypts for the small intestine. The top shows the mean number of labelled cells or mitoses per zone. The bottom shows the proportion of labelled cells or mitoses in the zones. ${ }^{\star} p<0.05,{ }^{\star} p<0.01$ versus $T P N+$ alanine.

determined. All tissues were scored blind (the person reading the slides was unaware of the group to which the slides belonged).

\section{STATISTICS}

All results are presented as the mean (SEM). Data were tested by a two sided $t$ test against the TPN controls. Mitotic data for the various sites for the TPN groups were subjected to a one way analysis of variance. In addition all the TPN data for the colon were subjected to a two way analysis of variance. The data were classified by two factors, site and glutamine. It is possible for one of these two factors to alter the effect of the other; this is measured by the interaction effect. If no interaction is seen, the test can be rerun without the interaction.

\section{Results}

Figure 1 shows the weights of the major regions of the gut; no effect of glutamine was seen in any site. The weights are expressed as a percentage of the final body weight, but the results were very similar if the uncorrected weights were considered. The weights of the small intestine, caecum, and colon were all significantly heavier in the orally fed group than in the TPN groups $(p<0.001)$.

There was no effect of any of the glutamine treatments on mitotic activity in the small intestine (fig 2), with the values for the TPN groups being significantly lower than in the orally fed animals $(\mathrm{p}<0.001)$.

In the colon there was an indication of increased mitotic activity with glutamine in all sites, which was significant at $\mathrm{p}=0.053$ in the mid colon. If all the TPN colon data were combined for a two way analysis, an effect of glutamine was seen at $\mathrm{p}=0.03$ (there was no interaction between glutamine and site). The values for the TPN groups were again also significantly lower than the orally fed animals $(\mathrm{p}<0.01)$.

Table 1 shows the results of the study of mitotic and labelled cells in histological sections. In the small intestine there were no 
Colon

Label distribution
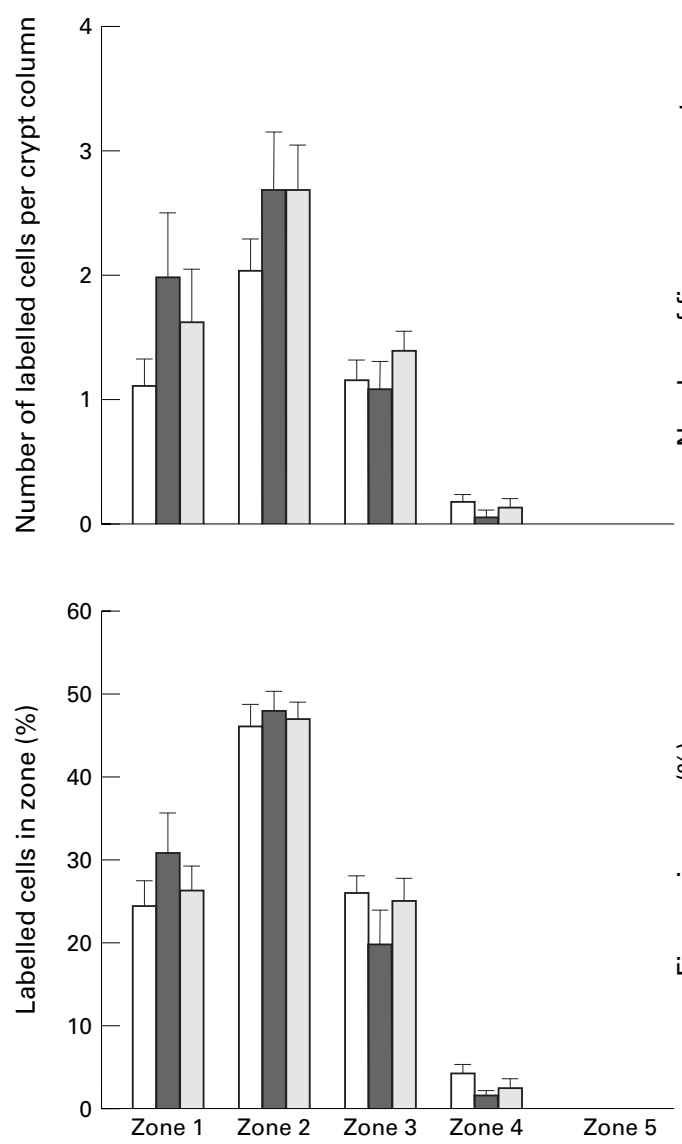

Mitoses distribution
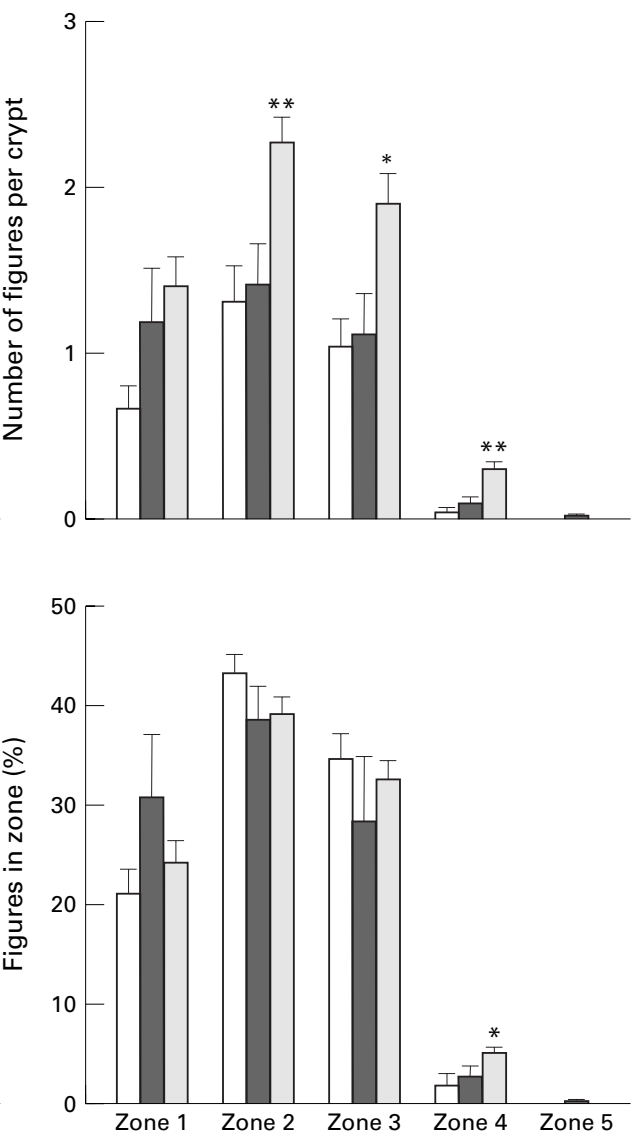

\section{$\square$ TPN + alanine \\ TPN + glutamine \\ Orally fed}

Figure 4 Data from sections reformatted to express the proportion of labelled cells within five equal zones of the crypt and the distribution of mitotic figures scored by superimposing a grid over the individual microdissected crypts for the colon. The top shows the mean number of labelled cells or mitoses per zone. The bottom shows the proportion of labelled cells or mitoses in the zones. ${ }^{\star} p<0.05,{ }^{\star *} p<0.01$ versus $T P N+$ alanine.

differences attributable to glutamine. However, the orally fed group had more labelled cells per crypt column $(p=0.05)$ and also had longer crypt columns $(p=0.027)$; thus the growth fraction of the orally fed rats was significantly reduced $(p=0.024)$. Note that there was no difference in labelling index as both the nominator and denominator had changed concurrently.

In the colon there seemed to be more mitoses and labelled cells per crypt column and this was reflected in a small but significant reduction in the growth fraction.

The data from sections were reformatted to express the number of labelled cells within five equal zones of the crypt. These data were also compared with the distribution of mitotic figures scored by superimposing a grid over the individual microdissected crypts. Figures 3 and 4 present the results of this zonal distribution for the small intestine and colon. The top half of the figures shows the mean number of labelled cells or mitoses per zone. The bottom half shows the proportion of labelled cells or mitoses in the zones. In the small intestine both measures showed that there was no difference attributable to glutamine, and that the orally fed rats had more labelled cells in zones 1 and 2 and more mitotic figures in zones $1,2,3$, and 4. When all the data were pooled there was no difference in the total number of mitotic or labelled cells between the two TPN groups, but the orally fed animals had significantly more labelled cells $(p=0.013)$ and mitoses $(p=0.002)$. When the small bowel data were used to look at the relative distribution of label, there were more labelled cells in zone 1 and less in zone 3 of the orally fed rats. There also seemed to be more labelled cells in zone 1 of the glutamine treated group $(\mathrm{p}=0.064)$.

In the colon there were no significant differences between groups for the labelling distributions; the mitotic data however showed several differences between the TPN and the orally fed animals in terms of absolute number of figures per zone. 
The pooled data showed that there were no significant differences in the total number of labelled cells in the colon, but there was a large increase in the total number of mitotic figures scored in the orally fed animals $(p=0.007)$.

When the distribution data were converted to relative distributions little effect was seen except for a slight increase in activity in zone 4 of the orally fed animals.

\section{Discussion}

Several studies have indicated that glutamine is one of the preferred fuel sources for the small intestine. In the large bowel the short chain fatty acid butyrate is the preferred fuel followed by glutamine then glucose. ${ }^{4}$ The results of this study show that although glutamine had some effects, especially on the colon, they were relatively modest. These effects could not just be attributable to the addition of more nutrients per se as there was no difference between the TPN group and the TPN group supplemented with $2 \%$ alanine. In many ways the equivocal nature of our results reflects the published literature: although some dramatic reports of the effects of glutamine have been published, many papers have also shown weak or little effects.

Some of this may reflect the use of several different models; especially pertinent is whether the normal intestine is studied, or that following TPN, elemental diet feeding, injury, or a combination of these factors. ${ }^{18}$ In our study we used rats that were relatively unstressed, and it is possible that the potential beneficial effects of glutamine may only become evident when body stores of glutamine are depleted by illness or injury. Energy requirements increase and may be modified following trauma ${ }^{6}$; thus the effects may only be apparent in such models of damage. Supplementation of TPN with glutamine has previously been reported not to improve gut barrier function or mucosal immunity in unstressed rats. ${ }^{19}$ In man treatment with growth hormone, glutamine, and a high carbohydrate-low fat diet for three weeks resulted in only modest improvements in electrolyte absorption and delayed gastric emptying, with no improvements in small bowel morphology, stool losses, or macronutrient absorption. ${ }^{20}$ Nonetheless, significantly improved survival in patients supplemented with glutamine has recently been reported in a six month study. ${ }^{21}$

Another relevant question is the choice of endpoint: thus although there may be a beneficial effect of glutamine, increased proliferation may not be the main factor; a general increase in protein synthesis ${ }^{22}$ or alterations in enterocyte transport function ${ }^{23}$ may be more important. This may be particularly pertinent with regard to reducing intestinal translocation of bacteria.

The consequence of temporal effects should also be borne in mind, as a few reports have indicated that increased proliferation may only be a transient effect. ${ }^{24}$

The discrepancies between the section based and squash based studies are of interest; one of the problems may be that conventional scoring techniques assume that the crypt is a cylinder with a hemispherical end. Savidge and colleagues $^{25}$ used a confocal microscopical technique to score intact crypts and did not find the "traditional" pattern of distribution of mitoses, which could be explained by the intact crypts being more "flask" shaped. The microdissection technique does not need any assumptions on crypt shape as the crypt is scored directly.

Comparison of the two techniques showed that the crypt squash technique can yield valid results when used to study changes in the distribution of proliferative activity; furthermore, the squash data were better able to detect the large differences between TPN and oral feeding seen by us in previous studies using the crypt cell production technique, where the magnitude of the differences was similar to that seen in the weight data of the present study. Thus, even without the amplification of dividing cells provided by vincristine, native mitoses per crypt is a method with greater resolution than BrdU labelling. Furthermore, the method is far easier and quicker to perform, does not need subsequent reanalysis to convert to "standardised" crypts, and does not make assumptions on the exact shape of the crypts. To summarise, one should stay with the crypt, the whole crypt, and nothing but the crypt. ${ }^{26}$

In conclusion, although glutamine has some modest effects on proliferation, these are unlikely to have much impact. Nevertheless, the effects of glutamine may be greater if the animal is depleted of glutamine by stress or trauma.

1 Goodlad RA, Wilson TJ, Lenton W, et al. Intravenous but not intragastric urogastrone-EGF is trophic to the intestine of parenterally fed rats. Gut 1987;28:573-82.

2 Buchman AL, Moukarzel AA, Bhuta S, et al. Parenteral nutrition is associated with intestinal morphologic and functional changes in humans. $\mathcal{F}$ Parenter Enteral Nutr functional changes

3 Roediger WE. Utilization of nutrients by isolated epithelial cells of the rat colon. Gastroenterology 1982;83:424-9.

4 Chapman MA, Grahn MF, Giamundo P, et al. New Chapman MA, Grahn MF, Giamundo P, et al. New
technique to measure mucosal metabolism and its use to map substrate utilization in the healthy human large bowel. Br F Surg 1993;80:445-9.

5 O'Dwyer ST, Smith RJ, Hwang TL, et al. Maintenance of small bowel mucosa with glutamine-enriched parenteral nutrition. F Parenter Enteral Nutr 1989;13:579-85

6 Newsholme E, Hardy G. Supplementation of diets with nutritional pharmaceuticals. Nutrition 1997;13:837-9.

7 Haque SM, Chen $\mathrm{K}$, Usui $\mathrm{N}$, et al. Alanyl-glutamine dipeptide-supplemented parenteral nutrition improves intestinal metabolism and prevents increased permeability in rats. Ann Surg 1996;223:334-41.

8 Garcia-Arumi E, Schwartz S, Lopez-Hellin J, et al. Addition of glutamine does not improve protein synthesis and jejunal mucosa morphology in non-hypercatabolic stress. Physiol Res 1995;44:233-9.

9 Klimberg VS, Souba WW, Dolson DJ, et al. Prophylactic glutamine protects the intestinal mucosa from radiation glutamine protects the intestin
injury. Cancer 1990;66:62-8.

10 Yagi M, Sakamoto K, Hasebe K, et al. Effect of a glutamineenriched diet on small bowel allograft during immunosuppressive therapy. Nutrition 1997;13:778-82.

1 Michail S, Mohammadpour H, Park JH, et al. Effect of glutamine-supplemented elemental diet on mucosal adaptation following bowel resection in rats. F Pediatr Gastroenterol Nutr 1995;21:394-8.

12 Vanderhoof JA, Blackwood DJ, Mohammadpour $\mathrm{H}$, et al. Effects of oral supplementation of glutamine on small intestinal mucosal mass following resection. $7 \mathrm{Am}$ Coll Nutr 1992;11:223-7.

13 Goodlad RA. Microdissection-based techniques for the determination of cell proliferation in gastrointestinal epithelium: application to animal and human studies. In: Celis JE, ed. Cell biology: a laboratory handbook. New York:

14 Goodlad RA, Lee CY, Wright NA. Cell proliferation in the small intestine and colon of intravenously fed rats: effects of
urogastrone-epidermal growth factor. Cell Prolif 1992;25: urogastrone-c. 
15 Goodlad RA, Wilson TG, Lenton W, et al. Intravenous but not intragastric urogastrone-EGF is trophic to the intestinal

16 Chwalinski S, Potten CS, Evans G. Double labelling with bromodeoxyuridine and $3 \mathrm{H}$-thymidine of proliferating cells in small intestinal epithelium in steady state and after irradiation. Cell Tissue Kinetics 1988;21:317-29.

17 Goodlad RA, Wright NA. Epithelial kinetics, control and consequences of alterations in disease. In: Whitehead $\mathrm{R}$, ed. Gastrointestinal and oesophageal pathology. Edinburgh Churchill Livingstone, 1995:97-116.

18 Scheppach W, Dusel G, Kuhn T, et al. Effect of L-glutamine and n-butyrate on the restitution of rat colonic mucosa after acid induced injury. Gut 1996;38:878-85.

19 Spaeth G, Gottwald T, Haas W, et al. Glutamine peptide does not improve gut barrier function and mucosal immudoes not improve gut barrier function and mucosal immu1993; 17:317-23.

20 Scolapio JS, Camilleri M, Fleming CR, et al. Effect of growth hormone, glutamine, and diet on adaptation in short-bowel syndrome: a randomized, controlled study. Gastroenterology 1997;113:1074-81.

21 Griffiths RD. Outcome of critically ill patients after supplementation with glutamine. Nutrition 1997;13:752-4.

22 Higashiguchi T, Hasselgren PO, Wagner K, et al. Effect of glutamine on protein synthesis in isolated intestinal epithelial cells. F Parenter Enteral Nutr 1993;17:307-14.

23 Sarac TP, Seydel AS, Ryan CK, et al. Sequential alterations in gut mucosal amino acid and glucose transport after $70 \%$ in gut mucosal amino acid and glucose transport

24 Wiren ME, Permert J, Skullman SP, et al. No differences in mucosal adaptive growth one week after intestinal resection in rats given enteral glutamine supplementation or deprived of glutamine. Eur F Surg 1996;162:489-98.

25 Savidge TC, Walker-Smith JA, Phillips AD. Novel insights into human intestinal epithelial cell proliferation in health and disease using confocal microscopy. Gut 1995;36:36974 .

26 Goodlad RA. The whole crypt and nothing but the crypt. Eur $\mathcal{F}$ Gastroenterol Hepatol 1992;4:1035-6. 